Highly Energetic Physical Processes and

Mechanisms for Emission from Astrophysical Plasmas

IAU Symposium, Vol. 195, 2000

P. C. H. Martens, S. Tsuruta, and M. A. Weber, eds.

\title{
Candidates for Optical Counterpart in the Field of PSR 1821-24 in M28
}

\author{
F. K. Sutaria \\ Inter-University Centre for Astronomy and Astrophysics, Pune, India
}

\begin{abstract}
The 3-ms, X-ray pulsar RX J1824.2-2R52P has been identified at the 2- $\sigma$ level as a possible X-ray counterpart of the radio pulsar PSR 1821-24. The present work uses archival HST data for M28 to set upper limits on the optical V-band magnitude of PSR 1821-24 / RX J1824.22R452P. The optical limit extends the multiwavelength observations for this source and would provide constraints for theoretical models of pulsar emission.
\end{abstract}

The isolated radio pulsar PSR 1821-24 has a high spin-down rate leading to rotational energy loss rate of $2.2 \times 10^{36} \mathrm{erg} \mathrm{s}^{-1}$. This source has been detected as a rotation powered, nonthermal emitter of X-rays, having a luminosity $\left.L_{x}\right|_{0.1-2.4 \mathrm{keV}}=1.6 \times 10^{33}(d / 5.1 \mathrm{kpc})^{2} \mathrm{erg} \mathrm{s}^{-2}$, by ROSAT HRI observations (Becker \& Trümper 1998, and references therein). While the relation between pulsed optical and X-ray or radio emission is unclear, in general, the pulsed optical and higher energy components can be either due to nonthermal acceleration of particles in the pulsar magnetosphere or due to emission from heated polar caps. Unpulsed optical emission can be attributed to thermal cooling of neutron stars. This implies a surface temperature of $T<10^{5} \mathrm{~K}$ for an old object like PSR 1821-24. Both pulsed and unpulsed components are indirect probes of the poorly known conditions in the neutron star interior and of the neutron star equation of state. Few old pulsars $\left(\tau \sim 10^{6}\right.$ years $)$ have been detected in optical wavelengths (Mignani 1998). Thus, an upper limit on the V-band magnitude of PSR 1821-24 would add one more data point to this list, and a confirmed detection would constrain the amount of nonthermal optical emission from the neutron star magnetosphere.

Details of the observation parameters and data analysis are given in Sutaria (in preparation). Briefly, the data was obtained from a Sept. 1997 HST-WFPC2 exposure of $1120 \mathrm{~s}$. The radio position of PSR 1821-24 was obtained from the dense radio-timing observation of Cognard et al. (1996; also see Table 1b). Accounting for the proper motion of the pulsar, the coordinates of PSR 1821-24 relative to the 1997 epoch are $\alpha=18^{h} 24^{m} 32.0052^{s}$ and $\delta=-24^{\circ} 52^{\prime} 10^{\prime \prime} \cdot 7212$. The absolute astrometric accuracy of WFPC2 is only $\simeq 0.5^{\prime \prime}$, mainly because of inaccuracies of $0.5^{\prime \prime}$ to $1^{\prime \prime}$ in the positions of stars used in the Guide Star Catalog. Taking a lower estimate of $0.5^{\prime \prime}$ uncertainty, at least 8 optical sources have been detected in this region. The photometry of these sources and their relative offsets has been quoted in Table 2 . Of the eight candidates within the $0.5^{\prime \prime}$ astrometric "error circle", stars 4 and 5 are too bright to be considered for pulsar candidates. In order to determine whether these objects are foreground objects or not, photometric analysis in another band would be required. If the optical emission 
were entirely thermal in nature and hence strongly age dependent, PSR 1821-24 would be too faint to be detected in this $1120 \mathrm{~s}$ exposure (Sutaria, in preparation). Even assuming that the optical component is enhanced by nonthermal emission, PSR 1821-24 is unlikely to be as bright as any of the other 6 sources in Table 2 because of its age and distance, thus implying that a visual band magnitude of 23.7 in the STMAG system is only an upper limit on the optical counterpart for this source.

Table 1. (a) A few relevant properties of PSR 1821-24. (b) The radio timing astrometry of PSR 1821-24.

\begin{tabular}{ccc|ccc}
\hline & $(\mathrm{a})$ & & \multicolumn{3}{c}{$(\mathrm{b})$} \\
Property & Units & Value & Property & Units & Value \\
\hline Period $\left(P_{0}\right)$ & $\mathrm{s}$ & 0.00305 & $\alpha(J 2000)$ & $h \cdot m \cdot s$ & $18^{h} 24^{m} 32^{s} .006$ \\
$\dot{\mathrm{P}}$ & $\mathrm{s} / \mathrm{s}$ & $1.161 \times 10^{-18}$ & $\delta(J 2000)$ & $\circ . \cdot^{\prime \prime}$ & $-24^{\circ} 52^{\prime} 10^{\prime \prime} 702$ \\
$\tau$ & $10^{6} \mathrm{yr}$ & 30 & $\mu_{\alpha}$ & $\mathrm{mas} / \mathrm{yr}$ & $-1.27 \pm 0.17$ \\
Distance & $\mathrm{kpc}$ & 5.1 & $\mu_{\delta}$ & $\mathrm{mas} / \mathrm{yr}$ & $+3.0 \pm 3$ \\
\hline \hline
\end{tabular}

Table 2. No. of counts for sources detected in a $0.5^{\prime \prime}$ field around the radio position of PSR 1821-24. The offsets are calculated relative to the radio position of PSR 1821-24 (1997 epoch). All detections are at the $3.5 \sigma$ level above the detection threshold.

\begin{tabular}{ccccccc}
\hline $\begin{array}{c}\text { Star } \\
\text { No }\end{array}$ & $\alpha_{2000}$ & $\delta_{2000}$ & \multicolumn{2}{c}{ Offsets } & Total & STMAG \\
\hline 1 & 31.9756 & 10.998 & 0.402 & -0.2768 & 388.44 & 22.6 \\
2 & 32.0079 & 11.155 & -0.0375 & -0.4338 & 175.80 & 23.5 \\
3 & 32.0159 & 11.155 & -0.1455 & -0.4338 & 144.533 & 23.7 \\
4 & 32.0054 & 10.892 & -0.0027 & -0.1708 & 12227.12 & 18.9 \\
5 & 32.0022 & 10.741 & 0.0405 & -0.0198 & 14374.56 & 18.7 \\
6 & 32.0158 & 10.728 & -0.144 & -0.0068 & 363.38 & 22.72 \\
7 & 32.0118 & 10.599 & -0.144 & 0.1222 & 168.93 & 23.5 \\
8 & 31.9987 & 10.293 & 0.08835 & 0.4282 & 1083.147 & 21.5 \\
\hline \hline
\end{tabular}

\section{References}

Becker, W., \& Trümper, J. 1999, A\&A, 341, 803

Cognard, I., Bourgois, G., Lestrade, J.-F., Biraud, F., Aubry, D., Darchy, B., \& Drouhin, J. P. 1996, A\&A, 311, 179

Mignani, R. P. 1998, in Frontiers Sci. Ser., 24, Neutron Stars and Pulsars: Thirty Years after the Discovery, eds. N. Shibazaki et al. (Tokyo: Universal Academy Press), 335

Sutaria, F. K., in preparation 